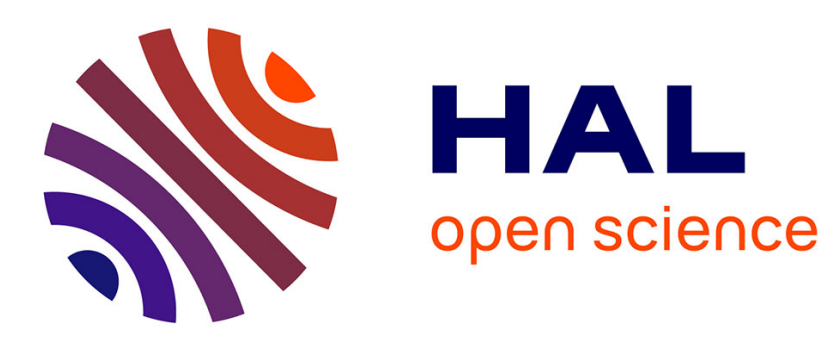

\title{
Set-membership Switched Observers based on Interval Characterization of the Estimation Error
}

\author{
Sara Ifqir, Vicenç Puig, Dalil Ichalal, Naïma Ait-Oufroukh, Saïd Mammar
}

\section{To cite this version:}

Sara Ifqir, Vicenç Puig, Dalil Ichalal, Naïma Ait-Oufroukh, Saïd Mammar. Set-membership Switched Observers based on Interval Characterization of the Estimation Error. 21st IFAC World Congress (IFAC 2020), Jul 2020, Berlin, Germany. pp.14261-14266, 10.1016/j.ifacol.2020.12.1165 . hal02877939

\section{HAL Id: hal-02877939 \\ https://hal.science/hal-02877939}

Submitted on 22 Jun 2020

HAL is a multi-disciplinary open access archive for the deposit and dissemination of scientific research documents, whether they are published or not. The documents may come from teaching and research institutions in France or abroad, or from public or private research centers.
L'archive ouverte pluridisciplinaire HAL, est destinée au dépôt et à la diffusion de documents scientifiques de niveau recherche, publiés ou non, émanant des établissements d'enseignement et de recherche français ou étrangers, des laboratoires publics ou privés. 


\title{
Set-membership Switched Observers based on Interval Characterization of the Estimation Error
}

\author{
Sara Ifqir* Vicenç Puig** Dalil Ichalal* \\ Naima Ait-Oufroukh* Saïd Mammar* \\ * IBISC Laboratory, Univ Evry, Paris-Saclay University, Evry, France. \\ (email: $\{$ sara.ifqir, dalil.ichalal, naima.aitoufroukh, \\ said.mammar\}@univ-evry.fr). \\ ** Institut de Robòtica i Informàtica Industrial (CSIC-UPC). Carrer \\ Llorens Artigas, 4-6, 08028 Barcelona.
}

\begin{abstract}
This paper presents a new set-membership estimation methodology for uncertain switched LPV discrete-time systems subject to unknown inputs, unmeasurable time-varying parameters and measurement noise. The proposed approach provides a guaranteed interval that is constructed as the sum of punctual state estimation and its corresponding estimation error limits. First, a punctual switched unknown input observer, robust against unknown inputs and time-varying uncertainties, is constructed. The proposed switched observer design is based on the solution of an optimization problem in terms of LMIs. Then, an outer-approximation of the enclosure set of state estimation error is computed using the admissible bounds of state and uncertainties. Application to vehicle state estimation is provided to show the design procedure and the flexibility of the proposed scheme. Comparison to real data demonstrates the accuracy and effectiveness of the obtained results.
\end{abstract}

\section{INTRODUCTION}

It is frequently necessary to estimate some variables describing the systems states that are not directly measurable for technical or economic reasons. This problem is solved by the use of "virtual sensors" generally called observers. State estimation has received considerable attention during the last decades [Baffet et al. 2009, Lin and Gao 2015, Yang and Wilde 1988]. Two distinct approaches have been proposed to estimate states variables. The first rests on conventional punctual estimators, such as Luenberger observers [Ciccarella et al. 1993], adaptive observers Kreisselmeier [1977], unknown inputs observers [Koenig et al. 2008] and sliding mode observers [Drakunov and Utkin 1995], where a specific value for the state is estimated at each time instant. However, due to the presence of uncertainties, the design of a conventional estimator that converges in the noisy-case towards the ideal value of the state, is often very challenging. The second method consists of using set-membership approaches [Alamo et al. 2005, Le et al. 2013a, Puig 2010] in order to estimate a set of admissible values (interval) for the state, and therefore, evaluate intuitively the accuracy of the estimation.

In this paper, a combination of both approaches is used. A new set-membership state estimation methodology, easy to implement, with less computing time is proposed for switched discrete-time LPV systems. The considered system is subject to unknown inputs, unmeasurable timevarying parameters and measurement noise. The proposed set-membership switched observer provides a guaranteed state interval which is constructed as the sum of punctual estimated trajectories and corresponding estimation error bounds.

The proposed switched observer can robustly estimate punctual state trajectories by decoupling unknown inputs and attenuating the effect of uncertainties. The necessary and sufficient conditions for the existence of such an observer are obtained with the aid of common quadratic Lyapunov function and Input-to-State stability technique. A constructive design procedure based on LMI conditions is given. Extra degrees of freedom provided by slack variables are used to reduce the conservativeness introduced by the use of the common Lyapunov function.

Since zonotopes utilize linear mappings and Minkowski sums which can be computed exactly and efficiently [Le et al. 2013b], a zonotopic threshold analysis method is used to compute an outer approximation of the reachable set of state estimation error. This characterized set allows to encompass all acceptable state estimation errors in a very simple and accurate way. The extent of this set informs us of the uncertainty with which the state vector is punctually estimated. Based on this approach, it is no longer necessary to use similarity transformations to design cooperative interval observers. Thus, the impulsive behavior as well as time computational complexity induced by these similarity transformations can be avoided. Finally, numerical simulations are provided to show the feasibility of the proposed scheme to estimate vehicle lateral velocity and yaw rate enclosure sets. Comparison to real data acquired using an instrumented vehicle demonstrates the potential of the estimation method. In fact, since the proposed strategies are simple to implement and do not require a huge real-time calculation, their application to industrial systems is suitable.

This paper is organized as follows: The problem statement is introduced in Section 3. Section 4 is devoted to present the main contribution of this work which is the design of 
switched unknown input observer for uncertain discretetime systems using a zonotopic bounding approach. Finally, in Section 5, an application of the proposed methodology to vehicle lateral velocity and yaw rate estimation illustrates the interest of such approach. Conclusions are presented in Section 6 .

Notation. In the rest of this paper, $\mathbb{R}\left(\mathbb{R}_{+}\right)$represents the set of all real (positive) numbers and $\mathbb{R}^{n}\left(\mathbb{R}_{+}^{n}\right)$ the $n$ dimensional real (positive) vector space. For a vector $x \in$ $\mathbb{R}^{n}$ or a matrix $M \in \mathbb{R}^{n \times n}$, one denotes $\bar{x}=\max \{0, x\}$, $\underline{x}=\bar{x}-x$ or $\bar{M}=\max \{0, M\}, \underline{M}=\bar{M}-M$. For two vectors $x_{1}$ and $x_{2}$, the inequalities $x_{1} \leq x_{2}\left(x_{1} \geq x_{2}\right)$ are interpreted element-wise. The relation $Q \succ 0$ (resp. $Q \prec 0$ ) means that the matrix $Q$ is positive (resp. negative) definite. $M^{T}$ stands for the transpose of the matrix $M$ and $I_{n}$ to the identity matrix of dimension $n \times n$.

\section{PRELIMINARIES}

As discussed in the Introduction, set-membership techniques are used in this paper. In this regard, it is necessary to introduce some basic definitions and operations used in zonotope and interval frameworks.

Definition 1. An unitary interval is denoted by $\mathbf{B}=$ $[-1 ; 1]$. An unitary box in $\mathbb{R}^{n_{x}}$, is a box composed of $n_{x}$ unitary intervals.

Lemma 1. [Efimov et al. 2013] Let the vector $x \in \mathbb{R}^{n_{x}}$ be a variable vector with given bounds $\bar{x}, \underline{x} \in \mathbb{R}^{n_{x}}$ such that $\underline{x} \leq x \leq \bar{x}$.

(1) If $A \in \mathbb{R}^{n_{x} \times n_{x}}$ is a constant matrix, then

$$
A^{+} \underline{x}-A^{-} \bar{x} \leq A x \leq A^{+} \bar{x}-A^{-} \underline{x} .
$$

(2) If $A \in \mathbb{R}^{n_{x} \times n_{x}}$ is a variable matrix such that $\underline{A} \leq$ $A \leq \bar{A}$ for some $\underline{A}, \bar{A} \in \mathbb{R}^{n_{x} \times n_{x}}$, then

$$
\begin{gathered}
\underline{A}^{+} \underline{x}^{+}-\bar{A}^{+} \underline{x}^{-}-\underline{A}^{-} \bar{x}^{+}+\bar{A}^{-} \bar{x}^{-} \leq A x \\
\leq \bar{A}^{+} \bar{x}^{+}-\bar{A}^{-} \underline{x}^{+}-\underline{A}^{+} \bar{x}^{-}+\underline{A}^{-} \underline{x}^{-}
\end{gathered} .
$$

where $\bullet^{+}=\max (0, \bullet)$ and $\bullet^{-}=\bullet^{+}-\bullet$.

Definition 2. [Le et al. 2013a] A zonotope of order $m$ in $\mathbb{R}^{n}$ is defined by the translation of the center $p \in \mathbb{R}^{n}$ of an unitary hypercube image of dimension $m$ in $\mathbb{R}^{n}$ under a linear transformation, the zonotope $\mathbb{X}$ is defined by:

$$
\mathbb{X}=\langle p, H\rangle=p \oplus H \mathbf{B}^{m}=\left\{p+H z: z \in \mathbf{B}^{m}\right\}
$$

Definition 3. The Minkowski sum of two sets $\mathbb{X}_{1}$ and $\mathbb{X}_{2}$ is given by $\mathbb{X}_{1} \oplus \mathbb{X}_{2}=\left\{x_{1}+x_{2}: x_{1} \in \mathbb{X}_{1}, x_{2} \in \mathbb{X}_{2}\right\}$.

Property 1. The Minskowski sum of two zonotopes $\mathcal{X}_{1}=$ $p_{1} \oplus H_{1} \mathbf{B}^{m_{1}}$ and $\mathcal{X}_{2}=p_{2} \oplus H_{2} \mathbf{B}^{m_{2}}$ is also a zonotope defined by $\mathcal{X}=\mathcal{X}_{1} \oplus \mathcal{X}_{2}=\left(p_{1}+p_{2}\right) \oplus\left[H_{1} H_{2}\right] \mathbf{B}^{m_{1}+m_{2}}$.

Definition 4. (Zonotope interval hull [Le et al. 2013a]). Consider the zonotope $\mathbb{X}=p \oplus H \mathbf{B}^{m}$, the smallest interval box that contains this zonotope, i.e. its interval hull, is computed by:

$$
\star \mathbb{X}=p \oplus \operatorname{rs}(H) \mathbf{B}^{n}
$$

where $r s(H)$ is a diagonal matrix such that $\operatorname{rs}(H)_{i i}=$ $\sum_{j=1}^{m}\left|H_{i j}\right|, i=1, \ldots, n$.

\section{PROBLEM STATEMENT}

Let us consider the following discrete-time LPV system

$$
\left\{\begin{array}{l}
x_{k+1}=\left(A_{0, \sigma(k)}+\Delta A_{\sigma(k)}\left(\xi_{k}\right)\right) x_{k}+ \\
\left(B_{0, \sigma(k)}+\Delta B_{\sigma(k)}\left(\xi_{k}\right)\right) u_{k}+E_{\sigma(k)} d_{k} \\
y_{k}=C x_{k}+\vartheta_{k}
\end{array}\right.
$$

where $x_{k} \in \mathbb{R}^{n_{x}}, u_{k} \in \mathbb{R}^{n_{u}}, d_{k} \in \mathbb{R}^{n_{d}}, y_{k} \in \mathbb{R}^{n_{y}}$, $\vartheta_{k} \in \mathbb{R}^{n_{\vartheta}}$ are the state, the control input, the unknown input, the measurement output and noise vectors, respectively. $\sigma(k): \mathbb{R}^{+} \rightarrow I=\{1,2, \ldots, N\}$ is the switching signal assumed to be available in real time. The matrices $A_{0, \sigma(k)} \in\left\{A_{0,1}, A_{0,2}, \ldots, A_{0, N}\right\}$ and $B_{0, \sigma(k)} \in$ $\left\{B_{0,1}, B_{0,2}, \ldots, B_{0, N}\right\}$ are the state space matrices assumed to be constant and known a priori. $E_{\sigma(k)} \in \mathbb{R}^{n_{x} \times n_{d}}$ and $C \in \mathbb{R}^{n_{y} \times n_{x}}$ stand for the unknown input distribution matrix and the output equation matrix, respectively. The matrices $\Delta A_{\sigma(k)}\left(\xi_{k}\right) \in\left\{\Delta A_{1}\left(\xi_{k}\right), \Delta A_{2}\left(\xi_{k}\right), \ldots, \Delta A_{N}\left(\xi_{k}\right)\right\}$ and $\Delta B_{\sigma(k)}\left(\xi_{k}\right) \in\left\{\Delta B_{1}\left(\xi_{k}\right), \Delta B_{2}\left(\xi_{k}\right), \ldots, \Delta B_{N}\left(\xi_{k}\right)\right\}$ are assumed to be unknown but bounded representing the uncertainty of the system caused by the unmeasurable time-varying parameter $\xi_{k} \in \Xi$, where $\Xi$ is an interval box given by:

$$
\Xi=\left\{\xi_{k} \in \mathbb{R}^{n_{\xi}} \mid \underline{\xi}_{k} \leq \xi_{k} \leq \bar{\xi}_{k}\right\}
$$

For easiness of further developments, the state space representation (5) is rewritten in the following equivalent form:

$$
\left\{\begin{array}{l}
x_{k+1}=A_{0, \sigma(k)} x_{k}+B_{0, \sigma(k)} u_{k}+E_{\sigma(k)} d_{k}+\delta_{\sigma(k)} \\
y_{k}=C x_{k}+\vartheta_{k}
\end{array}\right.
$$

where $\delta_{\sigma(k)}=\Delta A_{\sigma(k)}\left(\xi_{k}\right) x_{k}+\Delta B_{\sigma(k)}\left(\xi_{k}\right) u_{k}$.

For the system (7), a switched observer that reconstructs the state $x_{k}$ without any information on the unknown input $d_{k}$ can be constructed using the input $u_{k}$ and the measured output $y_{k}$ as

$$
\left\{\begin{array}{l}
\hat{x}_{k+1}=N_{\sigma(k)} \hat{x}_{k}+K_{\sigma(k)} y_{k}+G_{\sigma(k)} u_{k}-H_{\sigma(k)} y_{k+1} \\
\hat{y}_{k}=C \hat{x}_{k}
\end{array}\right.
$$

where the initial state $\hat{x}_{0} \in \mathbb{R}^{n_{x}} . N_{\sigma(k)}, K_{\sigma(k)}, G_{\sigma(k)}$ and $H_{\sigma(k)}$ are gain matrices with appropriate dimensions to be determined.

The dynamics of the state estimation error $e_{k}=x_{k}-\hat{x}_{k}$ is given by

$$
\begin{aligned}
e_{k+1}= & \left(I_{n}+H_{\sigma(k)} C\right) x_{k+1}-N_{\sigma(k)} \hat{x}_{k} \\
& -K_{\sigma(k)} y_{k}-G_{\sigma(k)} u_{k}+H_{\sigma(k)} \vartheta_{k+1}
\end{aligned}
$$

By denoting $P_{\sigma(k)}=I_{n}+H_{\sigma(k)} C$ and using (7), the dynamics of the state estimation error is

$$
\begin{aligned}
& e_{k+1}=\left(P_{\sigma(k)} A_{0, \sigma(k)}-K_{\sigma(k)} C\right) x_{k}-N_{\sigma(k)} \hat{x}_{k} \\
& +\left(P_{\sigma(k)} B_{0, \sigma(k)}-G_{\sigma(k)}\right) u_{k}+P_{\sigma(k)} E_{\sigma(k)} d_{k} \\
& +P_{\sigma(k)} \delta_{\sigma(k)}+H_{\sigma(k)} \vartheta_{k+1}-K_{\sigma(k)} \vartheta_{k}
\end{aligned}
$$

If the following relationships hold

$$
\begin{gathered}
N_{\sigma(k)}=P_{\sigma(k)} A_{0, \sigma(k)}-K_{\sigma(k)} C \\
P_{\sigma(k)} B_{0, \sigma(k)}-G_{\sigma(k)}=0 \\
P_{\sigma(k)} E_{\sigma(k)}=0
\end{gathered}
$$

then, (9) is reduced to

$$
e_{k+1}=N_{\sigma(k)} e_{k}+\Delta_{\sigma(k)}
$$

where $\Delta_{\sigma(k)}=\left[\begin{array}{llll}P_{\sigma(k)} & H_{\sigma(k)} & -K_{\sigma(k)}\end{array}\right]\left[\begin{array}{lll}\delta_{\sigma(k)} & \vartheta_{k+1} & \vartheta_{k}\end{array}\right]^{T}$.

From (11), one can see that $d_{k}$ has been decoupled under the conditions (10c), but the effect of uncertainties $\Delta_{\sigma(k)}$ still persists. Now, suppose that we know at each timeinstant a box, denoted $\left[e_{k}\right]$, which includes, with guarantee, all possible estimation error $e_{k}$, that is

$$
\forall \sigma(k), \forall k \geq 0, \quad e_{k} \in\left[e_{k}\right]=\left[\underline{e}_{k}, \bar{e}_{k}\right]
$$

where $\underline{e}_{k}$ and $\bar{e}_{k}$ are the lower and upper bounds of the box $\left[e_{k}\right]$. Accordingly, we can define a box at each instant 
$k$, denoted $\left[x_{k}\right]$, which contains in a guaranteed way the real value of the state vector $x_{k}$,

$$
\forall \sigma(k), \forall k \geq 0, \quad x_{k} \in\left[x_{k}\right]=\hat{x}_{k}+\left[e_{k}\right]
$$

So, an interval observer for the switched LPV system (7) can be compactly written as

$$
\begin{gathered}
\hat{x}_{k+1}=N_{\sigma(k)} \hat{x}_{k}+K_{\sigma(k)} y_{k}+G_{\sigma(k)} u_{k}-H_{\sigma(k)} y_{k+1} \\
{\left[x_{k}\right]=\hat{x}_{k}+\left[e_{k}\right]}
\end{gathered}
$$

Therefore, the proposed interval estimation method (14) rests on a combination between a punctual unknown input switched observer and a set-membership characterization of the estimation error. In fact, the punctual observer in (14a) generates an estimation of the nominal state trajectories. Afterwards, the effect of uncertainties on the estimated trajectories is addressed using interval analysis so that the real state vector is included in the box $\left[x_{k}\right]=$ $\left[\underline{x}_{k}, \bar{x}_{k}\right]$ defined by $(14 \mathrm{~b})$. This ensures that all the state trajectories consistent with parameter and initial state vectors uncertainties are enclosed by the interval observer (14) in a guaranteed way.

\section{SWITCHED UNKNOWN INPUTS OBSERVER DESIGN BASED ON INTERVAL ANALYSIS}

In this section, a constructive method to design the Switched Unknown Input Observer (14) for switched discrete-time systems (7) is presented. The robustness issue with respect to additive uncertainties $\delta_{\sigma(k)}$ is analyzed using Common Lyapunov function and Input-toState Stability principle. The design consists on finding the observer gain $K_{\sigma(k)}$ such that the observer error dynamics (11) is asymptotically stable when $\Delta_{\sigma(k)}=0$ and is ISSstable with respect to uncertainties when $\Delta_{\sigma(k)} \neq 0$.

\subsection{LMI formulation}

Consider the switched discrete-time linear system described by (7). The observer (14a) provides a punctual state estimation $\hat{x}_{k}$ if the observer gain $K_{\sigma(k)}$ is chosen such that the observer error dynamics is stable, and the effect of uncertainties is attenuated. To ensure that, let state first the following assumption.

Assumption 1. The necessary and sufficient conditions for the existence of the SUIO (8) for the system (7) are:

(1) $\operatorname{rank}\left(C E_{\sigma(k)}\right)=\operatorname{rank}\left(E_{\sigma(k)}\right)=q, \forall \sigma(k)$;

(2) The pair $\left(P_{\sigma(k)} A_{0, \sigma(k)}, C\right)$ is at least detectable $\forall \sigma(k)$.

Remark 1. The first condition in Assumption 1 ensures that equation (10c) is solvable, and a general solution can be given as follows:

$$
\begin{aligned}
H_{\sigma(k)}=-E_{\sigma(k)} & \left(C E_{\sigma(k)}\right)^{\dagger} \\
& +Y_{\sigma(k)}\left(I_{n}-\left(C E_{\sigma(k)}\right)\left(C E_{\sigma(k)}\right)^{\dagger}\right)
\end{aligned}
$$

where $\left(C E_{\sigma(k)}\right)^{\dagger}$ is the generalized inverse matrix of $C E_{\sigma(k)}$, given by

$$
\left(C E_{\sigma(k)}\right)^{\dagger}=\left(\left(C E_{\sigma(k)}\right)^{T}\left(C E_{\sigma(k)}\right)\right)^{-1}\left(C E_{\sigma(k)}\right)
$$

and $Y_{\sigma(k)}$ is an arbitrary matrix of appropriate dimension. For convenience of the notation, let define $U_{\sigma(k)}=$ $-E_{\sigma(k)}\left(C E_{\sigma(k)}\right)^{\dagger}$ and $J_{\sigma(k)}=I_{n}-\left(C E_{\sigma(k)}\right)\left(C E_{\sigma(k)}\right)^{\dagger}$, then (15) can be rewritten as

$$
H_{\sigma(k)}=U_{\sigma(k)}+Y_{\sigma(k)} J_{\sigma(k)}
$$

Remark 2. The second condition, sometimes referred as "strong detectability condition" is equivalent to that the transmission zeros from the unknown-input to the output must be stable, i.e.

$$
\operatorname{rank}\left(\left[\begin{array}{cc}
s I_{n}-A_{0, \sigma(k)} & E_{\sigma(k)} \\
C & 0
\end{array}\right]\right)=n+q,
$$

holds $\forall \sigma(k)$ and for all complex number $s$ with $\mathcal{R} e(s) \geq 0$.

The next theorem provides sufficient conditions for the state estimation error (11) to be ISS-stable under arbitrary switching. Before proceeding, we begin by stating the following equivalence.

Lemma 2. The following conditions are equivalent:

(1) There exists a symmetric matrix $Q$ such that

$$
\left[\begin{array}{cc}
N^{T} Q N+(\varepsilon-1) Q & N^{T} Q \\
Q N & Q-\gamma I_{n}
\end{array}\right] \prec 0
$$

(2) There exist a symmetric matrix $Q$ and a matrix $S$ such that

$$
\left[\begin{array}{ccc}
(\varepsilon-1) Q & 0 & N^{T} S^{T} \\
(*) & -\gamma I_{n} & S^{T} \\
(*) & (*) & -S^{T}-S+Q
\end{array}\right] \prec 0
$$

Proof. If we apply the Schur complement with respect to the block $(3,3)$ of $(20)$, we retrieve directly (19) by choosing $S=S^{T}=Q$, hence (1) implies (2). Moreover, from the first block of $(20)$, we have $(\varepsilon-1) Q \prec 0$. Then, multiplying (20) by $T=\left[\begin{array}{ccc}I_{n} & 0 & N^{T} \\ 0 & I_{n} & I_{n}\end{array}\right]$ on the left and $T^{T}$ on the right, we get (19), which establishes that (2) implies (1) and the proof is complete.

Theorem 1. The switched system (11) is uniformly ISSstable with respect to the switching signal $\sigma(k)$ if there exist a symmetric and positive definite matrix $Q$, matrices $S$ and $W_{i}, \forall i \in I$, a constant $\gamma>0$ for given positive scalars $\alpha$ and $0<\varepsilon<1$ such that the following condition holds

$$
\begin{gathered}
\min _{Q, S, W_{i}, X_{i}} \gamma \\
{\left[\begin{array}{ccc}
(\varepsilon-1) Q & 0 & A_{i}^{T}\left(I_{n}+U_{i} C\right)^{T} S^{T}+ \\
& & A_{i}^{T} C^{T} J_{i}^{T} X_{i}^{T}-C^{T} W_{i}^{T} \\
(*) & -\gamma I_{n} & S^{T} \\
(*) & (*) & -S^{T}-S+Q
\end{array}\right] \prec 0}
\end{gathered}
$$

with $W_{i}=S K_{i}$ and $X_{i}=S Y_{i}$ for any switching signal $\sigma(k)$. Furthermore, the state estimation error (11) is ultimately bounded and satisfies

$$
\lim _{k \rightarrow \infty}\left\|e_{k}\right\|_{2} \leq \sqrt{\frac{\gamma}{\alpha(1-\varepsilon)}}\left\|\Delta_{\sigma(k)}\right\|_{\infty}
$$

where $\Delta_{\sigma(k)}=P \delta_{\sigma(k)}$ and the maximum norm is given by $\left\|\Delta_{\sigma(k)}\right\|_{\infty}=\max \left\{\left|\Delta_{1}\right|,\left|\Delta_{2}\right|, \ldots,\left|\Delta_{N}\right|\right\}$.

Proof. For the stability analysis, we use the following common quadratic ISS-Lyapunov function

$$
V\left(e_{k}\right)=e_{k}^{T} Q e_{k}, \quad Q=Q^{T} \succ 0
$$

Setting $\Delta V\left(e_{k}\right) \triangleq V\left(e_{k+1}\right)-V\left(e_{k}\right)$, we have

$$
\Delta V\left(e_{k}\right)=e_{k+1}^{T} Q e_{k+1}-e_{k}^{T} Q e_{k}
$$

and using (11), we obtain

$$
\begin{aligned}
\Delta V\left(e_{k}\right)= & \left(N_{\sigma(k)} e_{k}+\Delta_{\sigma(k)}\right)^{T} Q\left(N_{\sigma(k)} e_{k}+\Delta_{\sigma(k)}\right)-e_{k}^{T} Q e_{k} \\
= & e_{k}^{T} N_{\sigma(k)}^{T} Q N_{\sigma(k)} e_{k}+\Delta_{\sigma(k)}^{T} Q N_{\sigma(k)} e_{k} \\
& +e_{k}^{T} N_{\sigma(k)}^{T} Q \Delta_{\sigma(k)}+\Delta_{\sigma(k)}^{T} Q \Delta_{\sigma(k)}-e_{k}^{T} Q e_{k}
\end{aligned}
$$


By adding the terms $\varepsilon e_{k}^{T} Q e_{k}$ and $-\gamma \Delta_{\sigma(k)}^{T} \Delta_{\sigma(k)}$, we have

$$
\begin{aligned}
& \Delta V\left(e_{k}\right)=\left[\begin{array}{c}
e_{k}^{T} \\
\Delta_{\sigma(k)}^{T}
\end{array}\right]\left[\begin{array}{cc}
N_{\sigma(k)}^{T} Q N_{\sigma(k)}+(\varepsilon-1) Q & N_{\sigma(k)}^{T} Q \\
(*) & Q-\gamma I_{n}
\end{array}\right] \\
& \times\left[\begin{array}{c}
e_{k} \\
\Delta_{\sigma(k)}
\end{array}\right]-\varepsilon e_{k}^{T} Q e_{k}+\gamma \Delta_{\sigma(k)}^{T} \Delta_{\sigma(k)}
\end{aligned}
$$

Then, for all $i \in I$, the satisfaction

$$
\left[\begin{array}{cc}
N_{i}^{T} Q N_{i}+(\varepsilon-1) Q & N_{i}^{T} Q \\
(*) & Q-\gamma I_{n}
\end{array}\right] \prec 0
$$

is equivalent to fulfill the following condition

$$
\left[\begin{array}{ccc}
(\varepsilon-1) Q & 0 & N_{i}^{T} S^{T} \\
(*) & -\gamma I_{n} & S^{T} \\
(*) & (*) & -S^{T}-S+Q
\end{array}\right] \prec 0, \quad \forall i \in I
$$

according to Lemma 2 . If we substitute $H_{i}$ given by (17) into $N_{i}$ in (10a), the matrix inequality in (26) becomes $\forall i \in I$

$$
\left[\begin{array}{ccc}
(\varepsilon-1) Q & 0 & A_{i}^{T}\left(I_{n}+U_{i} C\right)^{T} S^{T}+ \\
& & A_{i}^{T} C^{T} J_{i}^{T} Y_{i}^{T} S^{T}-C^{T} K_{i}^{T} S^{T} \\
(*) & -\gamma I_{n} & S^{T} \\
(*) & (*) & -S^{T}-S+Q
\end{array}\right] \prec 0
$$

By using $W_{i}=S K_{i}$ and $X_{i}=S Y_{i}$, LMI (21) is found. Then, under the conditions of the theorem, it follows from (24) that $\forall \sigma(k)$

$$
\Delta V\left(e_{k}\right)<-\varepsilon e_{k}^{T} Q e_{k}+\gamma \Delta_{\sigma(k)}^{T} \Delta_{\sigma(k)}
$$

that implies

$$
V\left(e_{k+1}\right)<(1-\varepsilon) V\left(e_{k}\right)+\gamma\left\|\Delta_{\sigma(k)}\right\|_{2}
$$

Integrating $(29)$ over the interval $[0, k)$, we have

$$
V\left(e_{k}\right) \leq(1-\varepsilon)^{k} V\left(e_{0}\right)+\gamma \sum_{m=0}^{k-1}(1-\varepsilon)^{k-m-1}\left\|\Delta_{\sigma(m)}\right\|_{2}
$$

knowing that (23) is satisfied for some $\beta>\alpha>0$, the following inequality

$$
\alpha\left\|e_{k}\right\|_{2} \leq V\left(e_{k}\right) \leq \beta\left\|e_{k}\right\|_{2}
$$

allows to deduce that

$$
\left\|e_{k}\right\|_{2} \leq \frac{1}{\sqrt{\alpha}}\left(\beta(1-\varepsilon)^{k}\left\|e_{0}\right\|_{2}^{2}+\frac{\gamma}{1-\varepsilon} \sum_{m=0}^{k-1}[1-\varepsilon]^{k-m}\left\|\Delta_{\sigma(m)}\right\|_{2}^{2}\right)^{\frac{1}{2}}
$$

Hence, when $k \rightarrow \infty$ and using the maximum norm of $\Delta_{\sigma(k)},(22)$ is obtained which ends the proof.

\subsection{Characterization of all possible estimation error}

In a bounded-error estimation context, we are interested in characterizing the interval of admissible values for the state consistent with the observed data, in the sense that the errors between model outputs and observations fall within prior bounds. This is done by recursively calculating a zonotopic outer-approximation of the effect of the parameter uncertainties and measurement noise. In this subsection, we show how to design a suitable interval observer under the following assumptions.

Assumption 2. The initial state $x_{0}$ is inside an initial zonotope $\mathbb{X}_{0}$, implying that the initial state estimation error $e_{0}$ is inside the zonotope $\mathbb{E}_{0}=C \mathbb{X}_{0}$, i.e. $e_{0} \in \mathbb{E}_{0}$.

Assumption 3. The measurement noise vector $\vartheta_{k}$ is unknown but bounded by a set

$$
V_{\vartheta}=\left\{\vartheta \in \mathbb{R}^{n_{\vartheta}}: \quad\left|\vartheta_{k}\right| \leq \mathcal{V}\right\}
$$

Assumption 4. It is assumed that the time variations of state and input vectors $x_{k}$ and $u_{k}$ are limited to some admissible bounds $\underline{x}_{k}, \bar{x}_{k}, \underline{u}_{k}$ and $\bar{u}_{k}$ such that $\underline{x}_{k} \leq x_{k} \leq$ $\bar{x}_{k}$ and $\underline{u}_{k} \leq u_{k} \leq \bar{u}_{k}$.

Note that since $\xi_{k} \in \Xi$, the system matrices $\Delta A_{\sigma(k)}\left(\xi_{k}\right)$ and $\Delta B_{\sigma(k)}\left(\xi_{k}\right)$ are bounded based on (6) as

$$
\begin{aligned}
& \underline{\Delta A}_{\sigma(k)} \leq \Delta A_{\sigma(k)}\left(\xi_{k}\right) \leq \overline{\Delta A}_{\sigma(k)} \\
& \underline{\Delta B}_{\sigma(k)} \leq \Delta B_{\sigma(k)}\left(\xi_{k}\right) \leq \overline{\Delta B}_{\sigma(k)}
\end{aligned}
$$

Therefore, using Assumption 4, the uncertain vector $\delta_{\sigma(k)}$ can be bounded by applying Lemma 1 . Subsequently, and under Assumption 3, the additive term $\Delta_{\sigma(k)}$ in (11) can be bounded by means of an interval box $\mathcal{D}$ given by:

$$
\begin{aligned}
& \mathcal{D}=\left\{\Delta_{\sigma(k)} \in \mathbb{R}^{n_{x}} \mid \Delta_{\sigma(k)} \leq \Delta_{\sigma(k)} \leq \bar{\Delta}_{\sigma(k)}, \underline{\Delta}_{\sigma(k)},\right. \\
& \left.\bar{\Delta}_{\sigma(k)} \in \mathbb{R}^{n_{x}}\right\}
\end{aligned}
$$

where

$$
\begin{aligned}
& \bar{\Delta}_{\sigma(k)}=P_{\sigma(k)}^{+} \bar{\delta}_{\sigma(k)}-P_{\sigma(k)}^{-} \underline{\delta}_{\sigma(k)}+\left(H_{\sigma(k)}-K_{\sigma(k)}\right) \mathcal{V} \\
& \underline{\Delta}_{\sigma(k)}=P_{\sigma(k)}^{+} \underline{\delta}_{\sigma(k)}-P_{\sigma(k)}^{-} \bar{\delta}_{\sigma(k)}+\left(H_{\sigma(k)}-K_{\sigma(k)}\right) \mathcal{V} \\
& \text { nd } \\
& \bar{\delta}_{\sigma(k)}=\overline{\Delta A}_{\sigma(k)}^{+} \bar{x}_{k}^{+}-\underline{\Delta A}_{\sigma(k)}^{+} \bar{x}_{k}^{-}-\overline{\Delta A}_{\sigma(k)}^{-} \underline{x}_{k}^{+}+\underline{\Delta A}_{\sigma(k)}^{-} \underline{x}_{k}^{-}+ \\
& \overline{\Delta B}_{\sigma(k)}^{+} \bar{u}_{k}^{+}-\underline{\Delta B}_{\sigma(k)}^{+} \bar{u}_{k}^{-}-\overline{\Delta B}_{\sigma(k)} \underline{u}_{k}^{+}+\underline{\Delta B}_{\sigma(k)}^{-} \underline{u}_{k}^{-} \\
& \underline{\delta}_{\sigma(k)}=\underline{\Delta A}_{\sigma(k)}^{+} \underline{x}_{k}^{+}-\overline{\Delta A}_{\sigma(k)}^{+} \underline{x}_{k}^{-}-\underline{\Delta A_{\sigma(k)}^{-}} \bar{x}_{k}^{+}+\overline{\Delta A}_{\sigma(k)}^{-} \bar{x}_{k}^{-}+ \\
& \quad \underline{B}_{\sigma(k)}^{+} \underline{u}_{k}^{+}-\overline{\Delta B}_{\sigma(k)}^{+} \underline{u}_{k}^{-}-\underline{\Delta B}-\bar{B}_{\sigma(k)}^{-} \bar{u}_{k}^{+}+\overline{\Delta B}_{\sigma(k)}^{-} \bar{u}_{k}^{-}
\end{aligned}
$$$$
\text { and }
$$

Furthermore, $\mathcal{D}$ can be rewritten as a zonotope $\mathcal{D}=\Delta_{c} \oplus$ $\mathcal{H}_{\bar{\Delta}_{\sigma(k)}} \mathbf{B}^{n}$, where $\mathcal{H}_{\bar{\Delta}_{\sigma(k)}} \in \mathbb{R}^{n_{x} \times n_{x}}$ is a diagonal matrix with the main diagonal being $\bar{\Delta}_{\sigma(k)}$ and $\Delta_{c}$ is a known and constant vector.

In this regard, the state estimation (11) can be bounded by the zonotope $e_{k+1} \in \mathbb{E}_{k+1}=\left\langle e_{k+1}^{c}, \mathcal{H}_{k+1}\right\rangle$ with

$$
\begin{gathered}
e_{k+1}^{c}=N_{\sigma(k)} e_{k}^{c} \\
\mathcal{H}_{k+1}=\left[\begin{array}{ll}
N_{\sigma(k)} & \mathcal{H}_{k} \\
\mathcal{H}_{\bar{\Delta}_{\sigma(k)}}
\end{array}\right]
\end{gathered}
$$

where $e_{k+1}^{c}$ and $\mathcal{H}_{k+1}$ are the center and segment matrix of the zonotope $\mathbb{E}_{k+1}$, respectively. The equivalent compact description of (36) is given as follows

$$
\mathbb{E}_{k+1}=N_{\sigma(k)} \mathbb{E}_{k} \oplus \mathcal{D}
$$

An interval for the estimation error $e_{k}$ can be obtained by computing the interval hull $\star \mathbb{E}_{k}$ (cf. Definition 4) of the zonotope $\mathbb{E}_{k}$.

\section{APPLICATION TO VEHICLE LATERAL DYNAMICS}

\subsection{Vehicle Model}

Vehicle lateral dynamics may be modeled using a two degree of freedom (2-DOF) model known as the "bicycle model" to describe the lateral and yaw motions Rajamani [2011].

Note that vehicles traveling at high speeds may be affected by lateral wind disturbances, resulting in lateral motion. The acting point of the resulting force, $f_{w}$, is called the aerodynamics center $(\mathrm{AC})$. The distance between the aerodynamics center and the center of gravity is noted by $l_{w}$. Therefore, the vehicle lateral motion when subject to wind force can be expressed using the following state space model:

$$
\left[\begin{array}{c}
\dot{v}_{y} \\
\ddot{\psi}
\end{array}\right]=A_{v}\left[\begin{array}{c}
v_{y} \\
\dot{\psi}
\end{array}\right]+B_{v} \delta_{f}+E_{v} f_{w}
$$

where 
$A_{v}=\left[\begin{array}{cc}\frac{-\left(c_{f}+c_{r}\right)}{m v_{x}} & \frac{c_{r} l_{r}-c_{f} l_{f}}{m v_{x}}-v_{x} \\ \frac{c_{r} l_{r}-c_{f} l_{f}}{I_{z} v_{x}} & -\frac{c_{r} l_{r}^{2}+c_{f} l_{f}^{2}}{I_{z} v_{x}}\end{array}\right], B_{v}=\left[\begin{array}{c}\frac{c_{f}}{m} \\ \frac{c_{f} l_{f}}{I z}\end{array}\right], E_{v}=\left[\begin{array}{c}\frac{1}{m} \\ \frac{l_{w}}{I_{z}}\end{array}\right]$

where $m, I_{z}$, are the mass and the yaw moment, $v_{x}$ and $v_{y}$ are lateral and longitudinal velocities, $\dot{\psi}$ is the yaw rate, $l_{f}, l_{r}$ are distances from front and rear axle to the center of gravity (CG). $c_{f}, c_{r}$ are the cornering stiffness of front and rear tires while $\delta_{f}$ represents the front steering angle. In the vehicle model (38), yaw rate $\dot{\psi}$ is available for measurement. Moreover, vehicle longitudinal velocity $v_{x}$ and cornering stiffness coefficients $c_{f}$ and $c_{r}$ are timevarying. In order to fully make use of the range variations of these uncertain parameters, the following representation is used:

$$
c_{f}=c_{f_{0}}+\Delta c_{f}, \quad c_{r}=c_{r_{0}}+\Delta c_{r}
$$

where the linear part, denoted by $c_{i 0}, \mathrm{i} \in\{r, f\}$, presents a priori known nominal value. The readjustment variable, i.e $\Delta c_{i}, i \in\{f, r\}$ assumed to be unknown but bounded, is added to correct the value of the cornering stiffness coefficients in case that a change occur in road adhesion or vehicle loading condition. Whereas, the longitudinal velocity is considered piecewise constant, and, different local sub-model are derived. A switching strategy that depends on the measured velocity is implemented.

By applying the first order Euler approximation on model (38) and taking into account the above consideration, a discrete-time model of the form (5) is obtained where, $\xi_{k}=\left[\Delta c_{f} \Delta c_{r}\right]^{T} \in \Xi$. Using the switched unknown input observer (14), the state estimate $\hat{x}_{k}$ is made robust against unknown lateral wind gusts disturbance and aforementioned uncertainties.

\subsection{Simulations results}

The real data used in the validation process are acquired using a prototype instrumented vehicle in a test track located in the city of Versailles-Satory, France. An artificial lateral wind gust input (Figure 1) is incorporated into the real measurements in simulation using nonlinear vehicle model [Rajamani 2011]. Steering angle and longitudinal velocity profiles are plotted in Figure 1. The simulation test is performed considering three local vehicle dynamics in which the longitudinal velocity is considered constant and differs from one mode to another. This can be seen as a subdivision of the range of variation of the longitudinal speed in three sub-regions, for low, medium and high speed. In the present scenario, the local longitudinal velocity values are: $v_{x}^{1}=8.5 \mathrm{~m} / \mathrm{s}, v_{x}^{2}=13.55 \mathrm{~m} / \mathrm{s}$ and $v_{x}^{3}=18.05 \mathrm{~m} / \mathrm{s}$. The switching law presented in Figure 2 is computed as in the earlier chapters as follows:

$$
\sigma(k)=\left\{\begin{array}{lll}
1 & \text { if } & v_{x} \in\left[V_{x}^{0}, V_{x}^{1}[\right. \\
2 & \text { if } & v_{x} \in\left[V_{x}^{1}, V_{x}^{2}[\right. \\
3 & \text { if } & v_{x} \in\left[V_{x}^{2}, V_{x}^{3}\right]
\end{array}\right.
$$

with $V_{x}^{0}=10.6 m . s^{-1}, V_{x}^{1}=11 m . s^{-1}, V_{x}^{2}=16 m . s^{-1}$ and $V_{x}^{3}=19.8990 \mathrm{~m} . \mathrm{s}^{-1}$. Numerical simulations were performed using Matlab software environment. The punctual switched unknown input observer is initialized at $\hat{x}_{0}=$ $\left[\begin{array}{ll}0 & 0\end{array}\right]^{T}$. According to the procedure described in Subsection 4.1 , the corresponding gain matrices of the UIO (8) for the lateral dynamics system (7) are designed ${ }^{1}$. The resulting

\footnotetext{
1 Details are omitted due to space limitation.
}
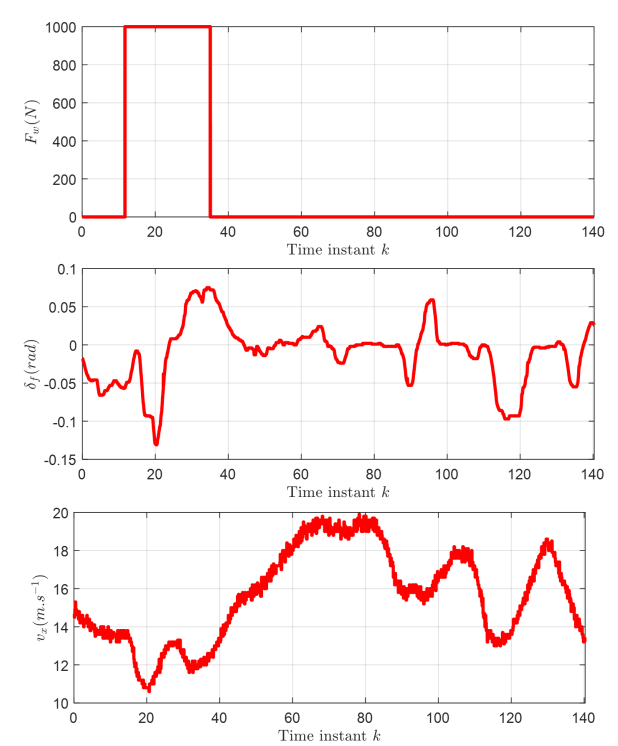

Fig. 1. Lateral wind gust input $f_{w}$, steering angle $\delta_{f}$, and longitudinal velocity $v_{x}$.

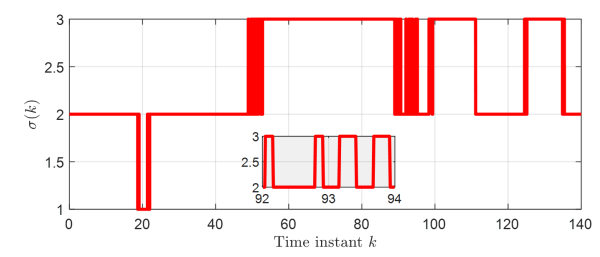

Fig. 2. Switching law $\sigma(k)$.

attenuation level is $\gamma=0.0120$ for $\varepsilon=0.5$ and a sampling time $T_{s}=0.6 s$.

Next, to implement the interval observer (14), the initial state is assumed to belong to the zonotope $\mathbb{X}_{0}=p_{0} \oplus H_{0} \mathbf{B}^{2}$, where $p_{0}=\left[\begin{array}{ll}0 & 0\end{array}\right]^{T}$ and $H_{0}=\left[\begin{array}{cc}0.01 & 0 \\ 0 & 0.01\end{array}\right]$. The upper and lower bounds of the state and input vector $u_{k}$ are defined by: $\bar{x}_{k}=-\underline{x}_{k}=\left[\begin{array}{ll}0.8 & 0.3\end{array}\right]^{T}, \bar{u}_{k}=u_{k}+0.01$ and $\underline{u}_{k}=u_{k}-$ 0.01. Furthermore, it is assumed that the cornering stiffness parameters are affected by $\pm 10 \%$ uncertainty in their nominal values. Thus, the box $\mathcal{D}$ can be easily deduced using (33). The estimation error bounds are computed at each sample time $k$ from the interval hull of the zonotope (37). The resulting bounds are then used to obtain the interval enclosing all possible values of the state vector $x_{k}$. Figure 3 compares the punctual estimated lateral velocity $\hat{v}_{y}$ and yaw rate $\hat{\dot{\psi}}$ with simulated and measured trajectories $v_{y}^{s}$ and $\dot{\psi}^{m}$, respectively. In Figure 4, the enclosure of all possible state estimation errors are shown. Finally, Figure 5 represents the guaranteed state estimation obtained for vehicle lateral velocity and yaw rate.

The proposed switched unknown input observer provides a successful estimation of the vehicle lateral dynamics. Particularly, the yaw rate is precisely estimated during steady-state and transient period when comparing to real measurement $\dot{\psi}^{m}$. Concerning lateral velocity, the performance of our observer is quite good expect when the steering angle changes quickly. In fact, with a quick steering movement of the driver and high lateral acceleration, lateral force increases significantly, and thus, reaches the nonlinear region. So, a linear approximation of lateral force 

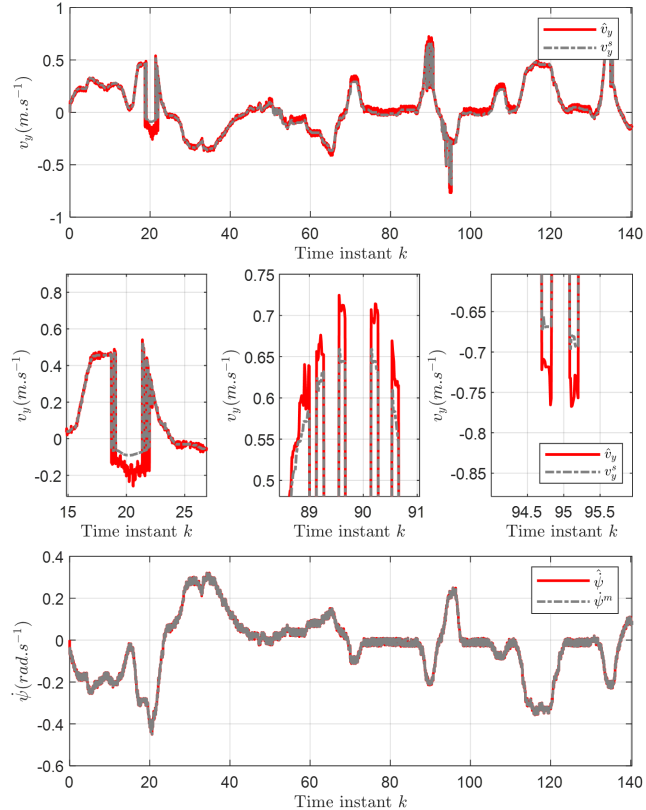

Fig. 3. Comparison of $v_{y}^{s}$ and $\hat{v}_{y}$ (fisrt two figures) and comparison of $\dot{\psi}^{m}$ and $\hat{\dot{\psi}}$.
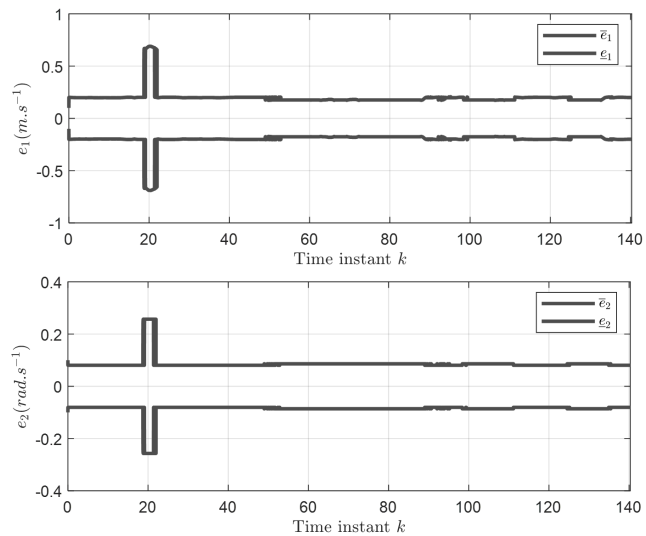

Fig. 4. Enclosure of $e_{1}=v_{y}^{s}-\hat{v}_{y}$ and $e_{2}=\dot{\psi}^{s}-\hat{\dot{\psi}}$.
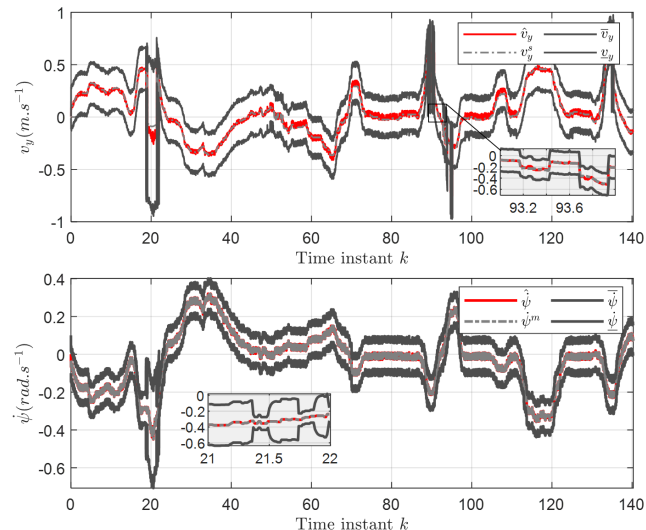

Fig. 5. Lateral velocity $v_{y}$ and yaw rate $\dot{\psi}$.

dynamics seems not so obvious to use since cornering stiffness coefficient cannot be identified with certainty. Therefore, the benefit of the adaptive dynamic tire model based estimator is evident. Admittedly, the proposed interval characterization of the estimation error allows to better understand and enhance observer performances, especially in the experiments where sudden change in road surface is expected. As shown in Figure 5, the simulated as well as measured data and their corresponding nominal estimates are inside the computed interval.

\section{CONCLUSION}

This paper investigates a set-membership state estimation scheme for uncertain switched discrete-time systems. The proposed methodology is based on a combination of punctual switched unknown input observers and zonotopic threshold analysis. It has been shown that the observer can be made robust against parameter uncertainties and measurement noise by using Input-to-State stability criteria. By characterizing the set of all possible state estimation errors, the worst-case residual bounds are computed using zonotopes. This guaranteed threshold avoids generating false alarms related to modeling errors and inaccurate measurements. An application to vehicle robust state estimation is presented providing satisfactory results.

\section{REFERENCES}

Alamo, T., Bravo, J.M., and Camacho, E.F. (2005). Guaranteed state estimation by zonotopes. Automatica, 41(6), 1035-1043.

Baffet, G., Charara, A., and Lechner, D. (2009). Estimation of vehicle sideslip, tire force and wheel cornering stiffness. Control Engineering Practice, 17(11), 12551264.

Ciccarella, G., Dalla Mora, M., and Germani, A. (1993). A luenberger-like observer for nonlinear systems. International Journal of Control, 57(3), 537-556.

Drakunov, S. and Utkin, V. (1995). Sliding mode observers. tutorial. In Proceedings of 1995 34th IEEE Conference on Decision and Control, volume 4, 33763378. IEEE.

Efimov, D., Raïssi, T., Chebotarev, S., and Zolghadri, A. (2013). Interval state observer for nonlinear time varying systems. Automatica, 49(1), 200-205.

Koenig, D., Marx, B., and Jacquet, D. (2008). Unknown input observers for switched nonlinear discrete time descriptor systems. IEEE Transactions on Automatic Control, 53(1), 373-379.

Kreisselmeier, G. (1977). Adaptive observers with exponential rate of convergence. IEEE transactions on automatic control, 22(1), 2-8.

Le, V.T.H., Stoica, C., Alamo, T., Camacho, E.F., and Dumur, D. (2013a). Zonotopes: From guaranteed stateestimation to control. John Wiley \& Sons.

Le, V.T.H., Stoica, C., Alamo, T., Camacho, E.F., and Dumur, D. (2013b). Zonotopic guaranteed state estimation for uncertain systems. Automatica, 49(11), 3418-3424.

Lin, J. and Gao, Z. (2015). Observers design for switched discrete-time singular time-delay systems with unknown inputs. Nonlinear Analysis: Hybrid Systems, 18, 85-99.

Puig, V. (2010). Fault diagnosis and fault tolerant control using set-membership approaches: Application to real case studies. International Journal of Applied Mathematics and Computer Science, 20(4), 619-635.

Rajamani, R. (2011). Vehicle dynamics and control. Springer Science \& Business Media.

Yang, F. and Wilde, R.W. (1988). Observers for linear systems with unknown inputs. IEEE transactions on automatic control, 33(7), 677-681. 